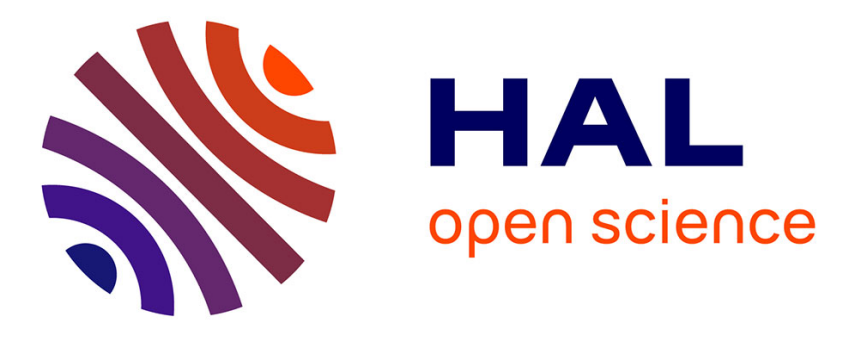

\title{
Non-negative Matrix Factorization under sparsity constraints to unmix in vivo spectrally resolved acquisitions
}

\author{
Anne-Sophie Montcuquet, Lionel Herve, Fabrice Navarro, Jean-Marc Dinten, \\ Jerome I. Mars
}

\section{To cite this version:}

Anne-Sophie Montcuquet, Lionel Herve, Fabrice Navarro, Jean-Marc Dinten, Jerome I. Mars. Nonnegative Matrix Factorization under sparsity constraints to unmix in vivo spectrally resolved acquisitions. WHISPERS 2010 - 2nd Workshop on Hyperspectral Image and Signal Processing: Evolution in Remote Sensing, Jun 2010, Reykjavik, Iceland. pp.30. hal-00625417

\section{HAL Id: hal-00625417 https://hal.science/hal-00625417}

Submitted on 21 Sep 2011

HAL is a multi-disciplinary open access archive for the deposit and dissemination of scientific research documents, whether they are published or not. The documents may come from teaching and research institutions in France or abroad, or from public or private research centers.
L'archive ouverte pluridisciplinaire HAL, est destinée au dépôt et à la diffusion de documents scientifiques de niveau recherche, publiés ou non, émanant des établissements d'enseignement et de recherche français ou étrangers, des laboratoires publics ou privés. 


\title{
NON-NEGATIVE MATRIX FACTORIZATION UNDER SPARSITY CONSTRAINTS TO UNMIX IN VIVO SPECTRALLY RESOLVED ACQUISITIONS
}

\author{
Anne-Sophie Montcuquet ${ }^{1,2}$, Lionel Hervé ${ }^{1}$, Fabrice Navarro ${ }^{1}$, Jean-Marc Dinten ${ }^{1}$, Jérôme I.Mars ${ }^{2}$ \\ ${ }^{1}$ CEA, LETI, MINATEC, 17 rue des martyrs, 38054 Grenoble cedex 9, France. \\ ${ }^{2}$ GIPSA-Lab, DIS, Rue de la houille blanche BP46, 38402 Grenoble, France
}

\begin{abstract}
Fluorescence imaging in diffusive media is an emerging imaging modality for medical applications which uses injected fluorescent markers (several ones may be simultaneously injected) that bind to specific targets, as tumors. The region of interest is illuminated with near infrared light and the emitted back fluorescence is analyzed to localize the fluorescence sources. To investigate thick medium, as the fluorescence signal decreases with the light travel distance, any disturbing signal, such as biological tissues intrinsic fluorescence - called autofluorescence -, is a limiting factor. To remove autofluorescence and isolate each specific fluorescent signal from the others, a spectroscopic approach, based on Non-negative Matrix Factorization, is explored. We ran an NMF algorithm with sparsity constraints on experimental data, and successfully obtained separated in vivo fluorescence spectra.
\end{abstract}

Index Terms - Fluorescence imaging, positive source separation, fluorescence in vivo spectroscopy.

\section{INTRODUCTION}

In fluorescence imaging, specific markers are injected to a patient, and bind specifically to targeted compounds, like tumors [1]. Several specific markers may be injected at once, and bind to different compounds or organs. The region of interest is illuminated with near infrared (NIR) light; an optimal wavelength range may be defined - between 600 and $900 \mathrm{~nm}$ - where the tissue absorption is lower and the marker optimally excited. Finally, the emitted back fluorescence signal is measured and the fluorescent source is localized. To investigate thick media for medical diagnostic application $(\simeq 4$ $\mathrm{cm}$ ), as the fluorescence signal gets exponentially weak with the light travel distance, the autofluorescence of tissues becomes a limiting factor. The analysis of a fluorescence signal impaired by autofluorescence may lead to a wrong localization of the markers: the signal needs to be preprocessed in order to remove autofluorescence. For in vivo fluorescence spectroscopy, the unmixing problem is referred to as a blind source separation problem since the spectra may vary according to the fluorescent dye biological environment. Fluores- cence spectra to separate are also supposed statistically dependent, which filters out many methods (such as ICA). Nonnegative Matrix factorization (NMF) differs from classical source separation methods (SVD, PCA) in that it forces the matrices factors to be non-negative, and thus suits to spectra separation. We propose to test this method on spectroscopic data, and to define a new regularized NMF algorithm constrained by sparsity.

\section{NON-NEGATIVE MATRIX FACTORIZATION}

For initial non-negative mixed data $M, \mathrm{NMF}$ proposes to find a couple of matrices $(A, S)$ with non-negatives coefficients, whose product optimally approaches $M$. The classical NMF definition says[2]:

Given a non-negative matrix $M \in R^{X \times Y}$, find non-negative matrices $A \in R^{X \times P}$ and $S \in R^{P \times Y}$ such that :

$M \simeq A S$

where non-negative matrices are matrices whose all factors are nonnegative and $P$ stands for the number of sources to unmix.

Applied to spectroscopy, matrix $A$ is considered as the weight vector, and $S$ as the spectra matrix, both containing as much elements $P$ as fluorescent sources to separate.

To find the particular matrices $A$ and $S$ that satisfy equation 1, two distinct steps must be considered. First, we need to define a criterion that link $M$ with $A$ and $S$. Then, in a second step, optimizing this criterion under the non-negativity constraint would lead to the best solution possible. Several criteria (the Euclidean distance, the Kullback-Leibler divergence [2]...) and different optimization methods (Alternating Least Square, update rules) may be chosen to obtain an NMF decomposition.

\section{SPARSITY CONSTRAINTS}

For local specific markers, we expect to get peaked weight columns in matrix $A$. A contrario, when unmixing is not 
complete, a residual autofluorescence baseline surrounds the specific marker peak. This property is depicted figure 1 .

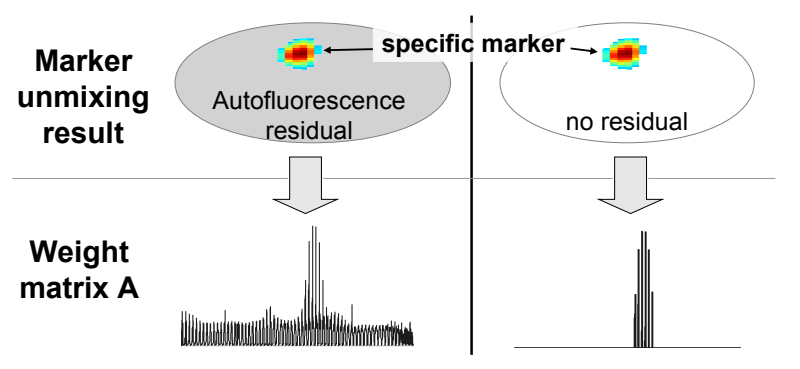

(a) Inaccurate unmixing $\quad$ (b) Correct unmixing

Fig. 1. Link between the quality of unmixing process and resulting weight matrix $A$.

An intuitive reasoning to improve unmixing would be to smooth over the unwanted autofluorescence residuals on specific markers weight profiles, by thresholding the smallest values: in other words, we look for sparse $A$ columns for specific markers contributions.

We introduce a sparsity value[3]: let us consider a weight matrix $A$ of size $X \times P$, the sparsity of a given column $A_{p}$ $(p \in(1, P))$ of $A$ is:

$$
\operatorname{sparsity}\left(A_{p}\right)=\frac{\sqrt{X}-\left(\sum_{x=1}^{X}\left|a_{x p}\right| / \sqrt{\sum_{x=1}^{X} a_{x p}^{2}}\right)}{\sqrt{X}-1}
$$

Sparsity value ranges from 0 for non-sparse results to 1 for extremely sparse results, as depicted figure 2 .

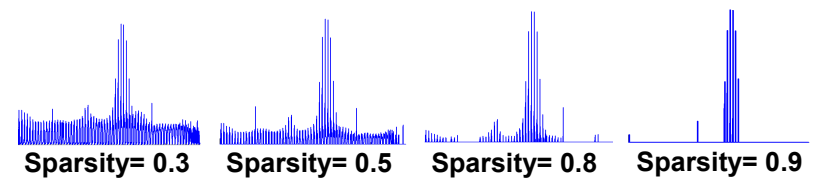

Fig. 2. Sparsity value ranges from 0 to 1 , from non-sparse to highly sparse signals.

In the next section, we propose an NMF algorithm that finds the perfect threshold so that our matrix A reaches a chosen sparsity value.

\section{SPARSITY-CONSTRAINED NMF ALGORITHM}

\subsection{Classical algorithm}

We first present a classical NMF algorithm without any constraint. In 2001, NMF popularity increased after Lee and Seung published two new NMF algorithm, based on the use of multiplicative update rules that minimize specific criteria.

\subsubsection{Cost function definition}

Different criteria to minimize, or cost functions, can be used [2]: we may cite the square of the euclidean distance between $M$ and $A S$, and the Kullback-Leibler divergence criterion. Here, we get interested in the cost function $F$, chosen as the square of the Euclidean distance between $M$ and $A S$ [2], lower bounded by 0 , and defined as:

$$
F=\sum_{x=1}^{N_{x}} N_{y}\left(m_{x y}-\sum_{p=1}^{P} a_{x p} s_{p y}\right)^{2}=\|M-A S\|^{2}
$$

\subsubsection{Optimization}

The following optimization problem is thus considered:

Problem 1 Minimize $F$ with respect to $A$ and $S$ subject to the constraints $A, S \geq 0$.

Lee and Seung proposed multiplicative update rules to solve Problem 1: those iterative rules offer a good compromise between speed and ease of implementation to solve the optimization problem (see problem 1); they are presented in the form of the following theorem:

Theorem 1 The distance $\|M-A S\|^{2}$ is non increasing under the update rules:

$$
S_{x p} \leftarrow S_{x p} \frac{\left(A^{t} M\right)_{x p}}{\left(A^{t} A S\right)_{x p}} \quad A_{p y} \leftarrow A_{p y} \frac{\left(M S^{t}\right)_{p y}}{\left(A S S^{t}\right)_{p y}}
$$

The proof of this theorem is given in Lee and Seung's publication [2].

We precisely got interested in those update rules because of their ease of implementation and speed, for which they were initially created.

\subsection{Constrained NMF}

In this section, taking the classical algorithm as a basis, we propose a new algorithm that takes into account sparsity constraints. The first NMF algorithm steps do not change from the classical one, but several sparsity steps are added for each iteration in order to reach a wanted sparsity value:

Algorithm: NMF with sparsity constraints

1. Initialize $A$ and $S$ with respectively positive constants and positive spectra models 
2. Update $S: S \leftarrow S \frac{\left(A^{t} M\right)}{\left(A^{t} A S\right)}$

3. Update $A: A \leftarrow A \frac{\left(M S^{t}\right)}{\left(A S S^{t}\right)}$

4. Each column $A_{p}$ of $A$ referring to weights of specific markers, for a wanted sparsity value spa for coefficients of column $A_{p}$, is changed into $\tilde{A}_{p}$ :

$\forall x \in(1, X)$,

$$
\tilde{a}_{x p}=\left\{\begin{array}{cl}
a_{x p} & \text { if } \quad a_{x p} \geq \frac{\max \left(a_{x p}\right)}{\beta_{p}} \\
0 & \text { otherwise }
\end{array}\right.
$$

with $\beta_{p}$ such as:

$$
\beta_{p}=\underset{\beta_{p}}{\operatorname{argmin}} \mid \operatorname{sparsity}\left(\tilde{A}_{p}\left(\beta_{p}\right)\right)-\text { spa } \mid
$$

5. restart steps 2 to 4 until stopping criterion is obtained (for example when $\|M-A S\|^{2}=\epsilon$ for a chosen $\epsilon$ ).

\section{RESULTS}

For in vivo experiments, an autofluorescence signal is necessarily measured. Then several specific markers may be used to simulate marked targets, such as tumors. In this section, we test NMF to unmix three overlapping different fluorescence sources, including the autofluorescence.

\subsection{Methods}

We perform feasibility experiments on a mouse. The animal procedure was in compliance with the guidelines of the European Union (regulation n86/609), taken in the French law (decree 87/848) regulating animal experimentation. All efforts were made to minimize animal suffering. The animal manipulation was performed with sterile techniques and approved by the Grenoble Animal Care and Use committee (France) (registration number 20_iRTSV Léti-FNG-02). An adult female nude mouse (Janvier, Le Genest saint-isle, France) was used throughout the experiments. It was housed in approved facilities, at $21 \pm 1 \mathrm{C}$ under diurnal lighting conditions. The mouse arrived at the animal facility two weeks before the experiments start and had free access to food and water.

To acquire spectrally resolved measurements, the animal is illuminated with a planar laser at $690 \mathrm{~nm}$. The emitted back fluorescence signal is collected along a line of $N_{x}$ points by a spectrometer coupled with a charge-coupled device camera (Andor Technologies): a $N_{x} \times N_{\lambda}$ acquisition is measured (see Figure 3). A translation stage, covering $N_{y}$ steps, is then used to get a scanning of the whole animal: $N_{y}$ acquisitions are obtained, each of size $N_{x} \times N_{\lambda}$. Before to run the NMF algorithm, our data of size $\left(N_{y} \times N_{x} \times N_{\lambda}\right)$ are reordered as a 2-D array of size $\left(N_{y} \times N_{x}, N_{\lambda}\right)$.

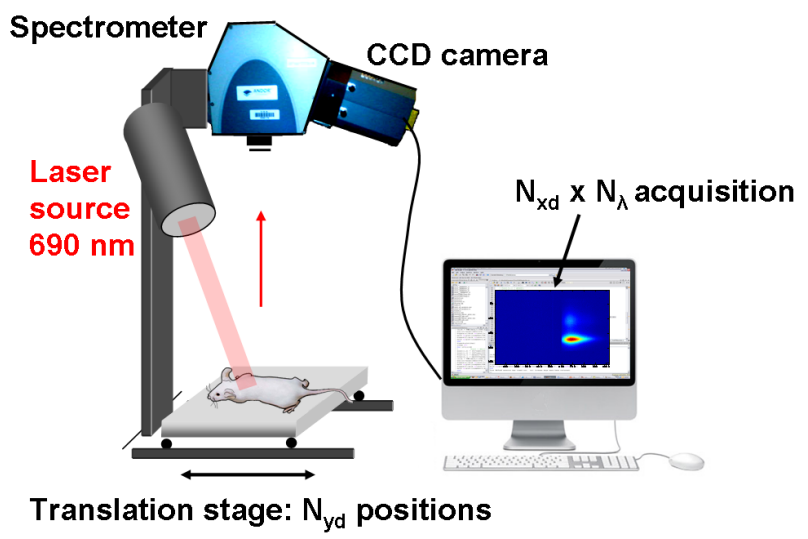

Fig. 3. Experimental set-up: acquisition on the animal and data processing.

To draw a parallel between acquisitions with or without specific fluorescence, a first acquisition of the animal is run as a reference, without any specific markers inserted (Figure 4-a). Then, several specific markers are added to simulate marked targets. First, a unique glass capillary tube filled with a specific marker is inserted subcutaneously on the animal, to classically simulate a marked target. The chosen marker is here $5 \mu \mathrm{l}$ of Indocyanine Green loaded into Lipid Nanoparticules (ICG-LNP) [4] at $0.35 \mu \mathrm{mol} / \mathrm{l}$. We will use this first example to illustrate the impact of sparsity constraints, running the algorithm with different wanted sparsity values.

Subsequently, a second capillary tube filled with $5 \mu$ l of Alexa 750 (an other specific marker) at $0.1 \mu \mathrm{mol} / 1$ is inserted. Three distinct fluorescent sources - autofluorescence, ICGLNP and Alexa 750 - whose emission spectra are overlapping have now to be unmixed. The resulting intensity scanning are presented Figure 4-b and Figure 4-c. In this precise example, we chose to constrain the sparsity level to 0.9 for the specific markers - ICG-LNP and Alexa 750 - weight matrices.

\subsection{Results}

We run the NMF algorithm on our data. For the first experiment, with a unique capillary tube (see data Figure 4-b), we analyze the sparsity constraint effect on results, by increasing the chosen value spa(see 4.2).

The number of components equal to zero in the column corresponding to ICG-LNP in matrix $A$ increases with sparsity value. Moreover, the spectra of matrix $S$ get closer to initializations with sparsity value increasing: $S$ was here initialized on purpose with mean spectra of considered data. Spectra of 


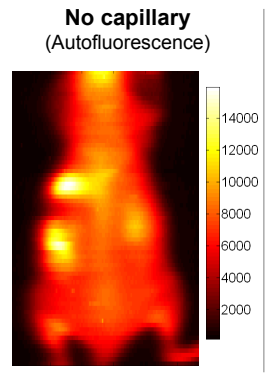

(a)

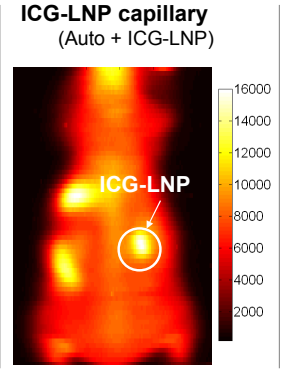

(b)

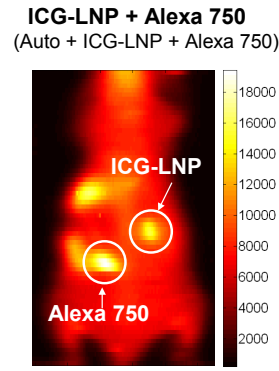

(c)

Fig. 4. (a) Autofluorescence acquisition (b) ICG-LNP tube inserted (c) ICG-LNP and Alexa 750 tubes inserted.

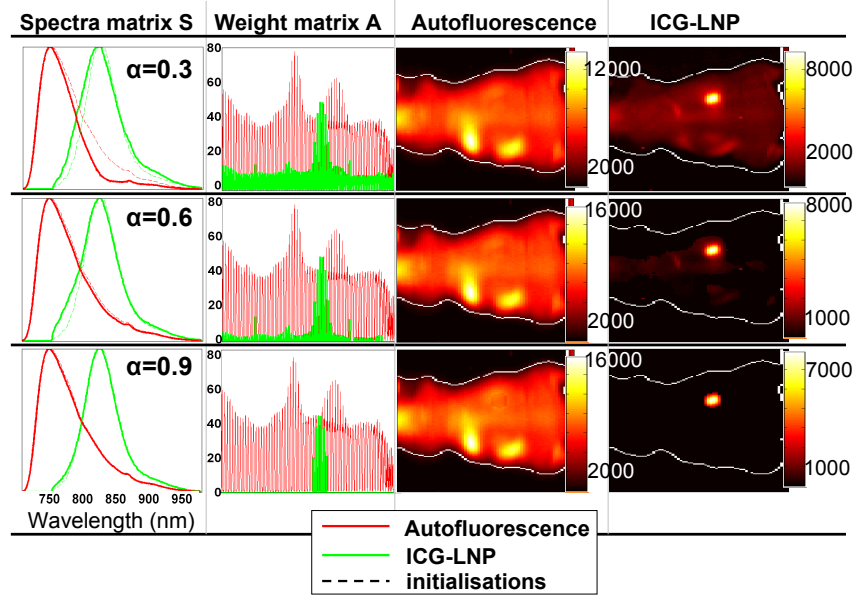

Fig. 5. FMN results: matrix $S$, matrix $A$, autofluorescence and ICG-LNP separated contributions for increasing values of wanted sparsity spa.

matrix $S$ getting closer to initialization may mean that results improve. For now, it is just an empirical observation to confirm. Finally, as expected, a strong sparsity value allows to remove the autofluorescence residuals and improves results (see Figure 5).

Figure 6 presents unmixing results of the second experiment (with two capillary tubes, initial data presented on Figure 4-c), for an high value of sparsity $(s p a=0.9)$. NMF under sparsity constraints successfully unmixed the three components. Nevertheless, a few errors may be noticed on the autofluorescence contributions: holes are present on ICG-LNP and Alexa 750 signals locations.

\section{CONCLUSION AND FUTURE WORK}

Beyond the specific fluorescence signal of specific markers used in optical imaging, the autofluorescence of biological tissues is also detected in the wavelength range we use, and needs to be removed to get accurate detection results. To un-

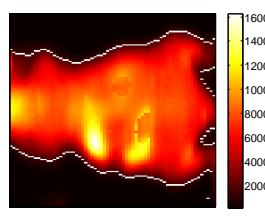

(a) Autofluo

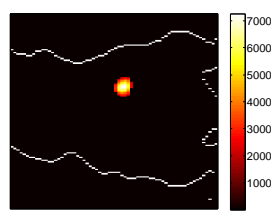

(b) ICG-LNP

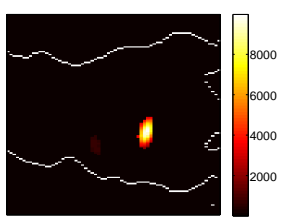

(c) Alexa 750
Fig. 6. Two capillary tubes experiment: unmixing results

mix fluorescence spectra, a blind source separation method was chosen: the Non-negative Matrix Factorization. We presented a classical NFM algorithm, before to introduce sparsity constraints. The constrained NMF algorithm was run on in vivo acquisitions, and successfully unmixed up to three different fluorescent sources. Empirically, results seem to improve with sparsity value imposed to specific markers weights profiles, but we still have to prove the convergence of the constrained algorithm. Moreover, sparsity value is by now empirically chosen: future work will explore methods to predefine the best sparsity value to use, and improve the existent algorithm. As optical imaging tries to detect deeper and deeper embedded tumors, NMF - as a preprocessing tool to remove autofluorescence signal and isolate specific fluorescence contributions, sharpened by several constraints - is an helpful tool.

\section{REFERENCES}

[1] V. Ntziachristos, E.A. Schellenberger, J. Ripoll, D. Yessayan, E. Graves, A. Bogdanov, L. Josephson, and R. Weissleder, "Visualization of antitumor treatment by means of fluorescence molecular tomography with an annexin v-cy5. 5 conjugate," Proceedings of the National Academy of Sciences, vol. 101, no. 33, pp. 12294, 2004.

[2] D.D. Lee and H.S. Seung, "Algorithms for non-negative matrix factorization," Advances in neural information processing systems, vol. 13, pp. 556-562, 2001.

[3] P.O. Hoyer, "Non-negative matrix factorization with sparseness constraints," The Journal of Machine Learning Research, vol. 5, pp. 1457-1469, 2004.

[4] F. P. Navarro, M. Berger, M. Goutayer, S. Guillermet, V. Josserand, P. Rizo, F. Vinet, and I. Texier, "A novel indocyanine green nanoparticle probe for non invasive fluorescence imaging in vivo," Progress in biomedical optics and imaging, vol. 10, no. 30, pp. 1-10, 2009. 